\title{
Super-resolution imaging of a dielectric microsphere is governed by the waist of its photonic nanojet
}

\author{
Hui Yang†, Raphaël Trouillon, Gergely Huszka and Martin A.M. Gijs* \\ Laboratory of Microsystems, École Polytechnique Fédérale de Lausanne, 1015 Lausanne, \\ Switzerland
}

\section{S1. Numerical simulation of light propagation}

Mie theory provides an analytical solution to the problem of the diffraction of a plane wave by a homogeneous sphere or an infinite cylinder. In order to take into account a realistic description of complex geometries and materials, a number of computational electrodynamics modeling techniques have been developed. Studies have shown that the finite element method (FEM) has merits for investigating spherical surfaces, since its tetrahedral adaptive meshing algorithm allows smoothly following curved contours, thereby providing a good spatial resolution and quantitative agreement with the Mie theory solution ${ }^{1-3}$. This feature makes FEM a promising modeling technique that is suitable to investigate near-field electromagnetic solutions and to reconcile theory and experiment.

The numerical study of the light propagation through the barium titanate glass microsphere (refractive index $n_{m s}=1.68$ ) and surrounding water medium ( $n_{w}=1.33$ ) was carried out by FEM in COMSOL Multiphysics software. A scalar equation was used to study transverse electric waves in a two-dimensional model,

$$
\nabla \times\left(\mu_{\mathrm{r}}^{-1} \nabla \times \boldsymbol{E}\right)-\left(\varepsilon_{\mathrm{r}}-\mathrm{j} \kappa / \omega \varepsilon_{0}\right) k_{0}^{2} \boldsymbol{E}=0
$$

where $\mu_{r}$ is the relative permeability, $\varepsilon_{r}$ is the relative permittivity, $\varepsilon_{0}$ is the vacuum permittivity, $\kappa$ is the electric conductivity, $\omega$ is the angular frequency and $k_{0}$ is the free-space wave number. In this model, the scattering boundary condition is used at all exterior boundaries, and the continuity boundary condition is used at material interfaces. A light source with wavelength of $600 \mathrm{~nm}$ and having the same width as the diameter of the microsphere was placed far away from the frontsurface of the microsphere. During meshing, an element size of $\sim 22 \mathrm{~nm}$ was obtained, i.e. $\sim$ one thirtieth of the wavelength, which was sufficiently small to obtain a precise solution. After the model was solved, the light intensity distribution was obtained by multiplying the electric field by its complex conjugate. 


\section{S2. Generation of the photonic nanojet}

A FEM study of the electromagnetic wave propagation through a $6 \mu \mathrm{m}$ microsphere in water medium is shown in Figure S1. For comparison, a light source with length half of the microsphere diameter is placed on the left or right side of the microsphere, but far away from the front-surface of the microsphere. Most illumination light is refracted on the front-surface and refracted again when exiting from the rear-surface of the microsphere. Light sources placed above the left half (Figure S1(a)) and above the right half of the microsphere (Figure S1(b)), respectively, show mirrored light intensity distributions. When a full light source, i.e. having the same width as the microsphere, is applied (Figure S1(c)), the wave-vector components $k \|$ originating from the left and right part locally eliminate each other by destructive interference, resulting in a nanojet that can maintain a highly confined transverse beam width and a FWHM that can be sub-wavelength. At the same time, the wave-vector components $\mathrm{k} \perp$ are superimposed and the photonic nanojet can extend more than $2 \lambda$ beyond the sphere along the wave propagation direction. Therefore, the photonic nanojet is a direct result of interference effects of the optical fields. 
(a) half light source on the left

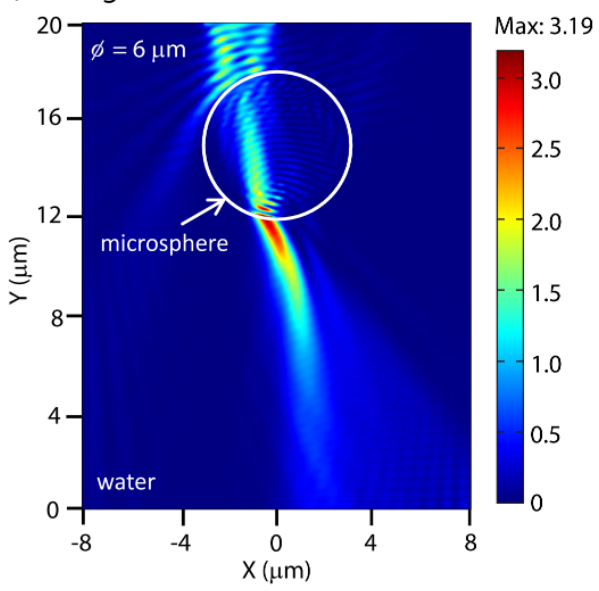

(c)

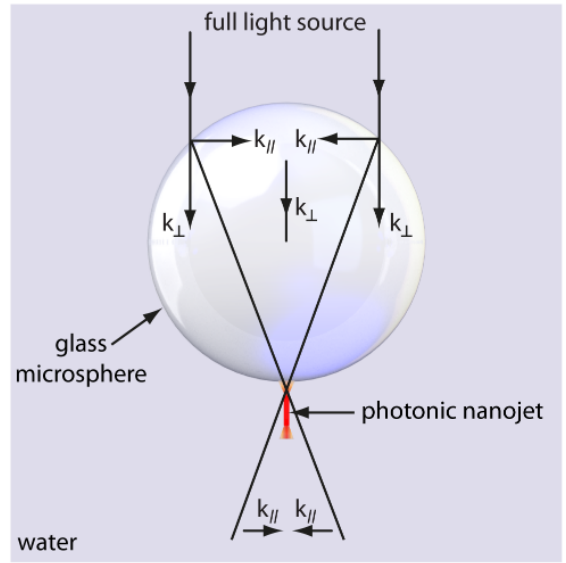

(b) half light source on the right

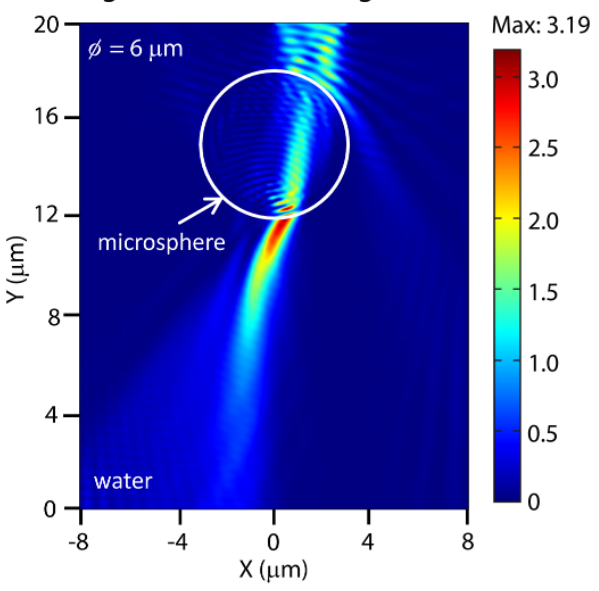

(d) full light source

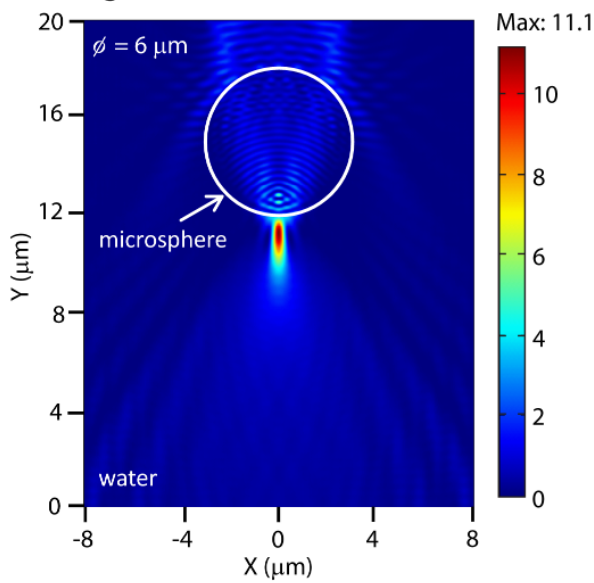

Figure S1. Mechanism of nanojet generation. (a,b) FEM simulation of the light propagation through a $6 \mu \mathrm{m} \varnothing$ barium titanate microsphere in water. A light source with a size half of the microsphere diameter is placed on the left side (a) and the right side (b) of the microsphere but far away from its front-surface. (c) Concept of the nanojet generation. When the microsphere is illuminated by a full light source, the left and right wave-vectors $k \|$ are canceling each other, while the $\mathrm{k} \perp$ wave vectors are superimposed. Interference of the optical fields therefore can generate the nanojet with confined transverse beam width and extended longitudinal beam length. (d) FEM simulation of the light propagation through a $6 \mu \mathrm{m} \varnothing$ barium titanate microsphere in water with a light source having the same size as the microsphere diameter. A photonic nanojet with sub-diffractional transverse beam width is generated indeed near the rear-surface of the microsphere.

\section{S3. Comparison between incoherent and coherent light source in the FEM study}

In order to evaluate the influence of using an incoherent illumination source or a coherent light source for the numerical analysis results, we performed a FEM study on 11 models with light source at different positions to mimic the incoherent illumination. In these models, the $6 \mu \mathrm{m} \varnothing$ microsphere 
is immersed in water, and an electromagnetic wave with wavelength of $600 \mathrm{~nm}$ is applied on a boundary far away from the top-surface of the microsphere. The boundary in these 11 models is placed on a position with distance to the microsphere's top-surface varying over a region $(-\lambda / 2, \lambda / 2)$. The boundary in the model shown in Figure 1(b) in the manuscript is set as the original place on the vertical axis, i.e. $\mathrm{y}_{0}=0$. Assuming that the distance between the boundary in the other 10 models and the one in Figure $1(\mathrm{~b})$ is $\Delta \mathrm{y}=\mathrm{y}_{\mathrm{n}}-\mathrm{y}_{0}$, where $\mathrm{y}_{0}$ is the reference position in the initial simulations shown in Figure 1 and $y_{n}$ is the new reference position on the $y$-axis used in these phase shift simulations so that $\Delta y=-31 \lambda / 64,-7 \lambda / 16,-\lambda / 4,-3 \lambda / 32,-1 \lambda / 32,+\lambda / 16,+5 \lambda / 32,+11 \lambda / 32,+15 \lambda / 32$, $+\lambda / 2$. These position shifts introduce a phase shift.

After all the 11 models were solved, the light intensity distributions obtained from each model are superimposed, and a figure indicating the photonic nanojet under an incoherent-like illumination is therefore obtained, as shown in Figure S2(a). As a comparison, the FEM simulation with a coherent illumination source is shown in Figure S2(b). In Figure S2(a), the intensity scale bar showing the light intensity is obtained by averaging the values from the 11 solved models. Moreover, we further compare the intensity distribution along the lateral and vertical direction of the photonic nanojet in Figure S2(a) and S2(b). As indicated in Figure S2(c), the lateral intensity distribution is obtained along the $\mathrm{x}$-axis and its origin is put on the point with maximum intensity in the nanojet; the vertical intensity profile is plotted along the $\mathrm{y}$-direction where $\mathrm{x}=0$, and the origin is at the bottom-surface of the microsphere. Figure S2(d, e) show the lateral and vertical intensity profiles that are obtained from the incoherent illumination source and Figure S2(f, g) show the profiles obtained from the coherent source. As illustrated in Figure S2(d) and S2(f), the intensity profile along the nanojet waist doesn't significantly change when using an incoherent or a coherent light source in the FEM study, indicating that our simulation method can provide sufficient information to compare with the pointspread-function obtained from the experimental results. Comparing Figure S2(e) and S2(g), the intensity of the nanojet decays faster along the vertical axis under coherent light, this result however won't change the resolution of the imaging system. 

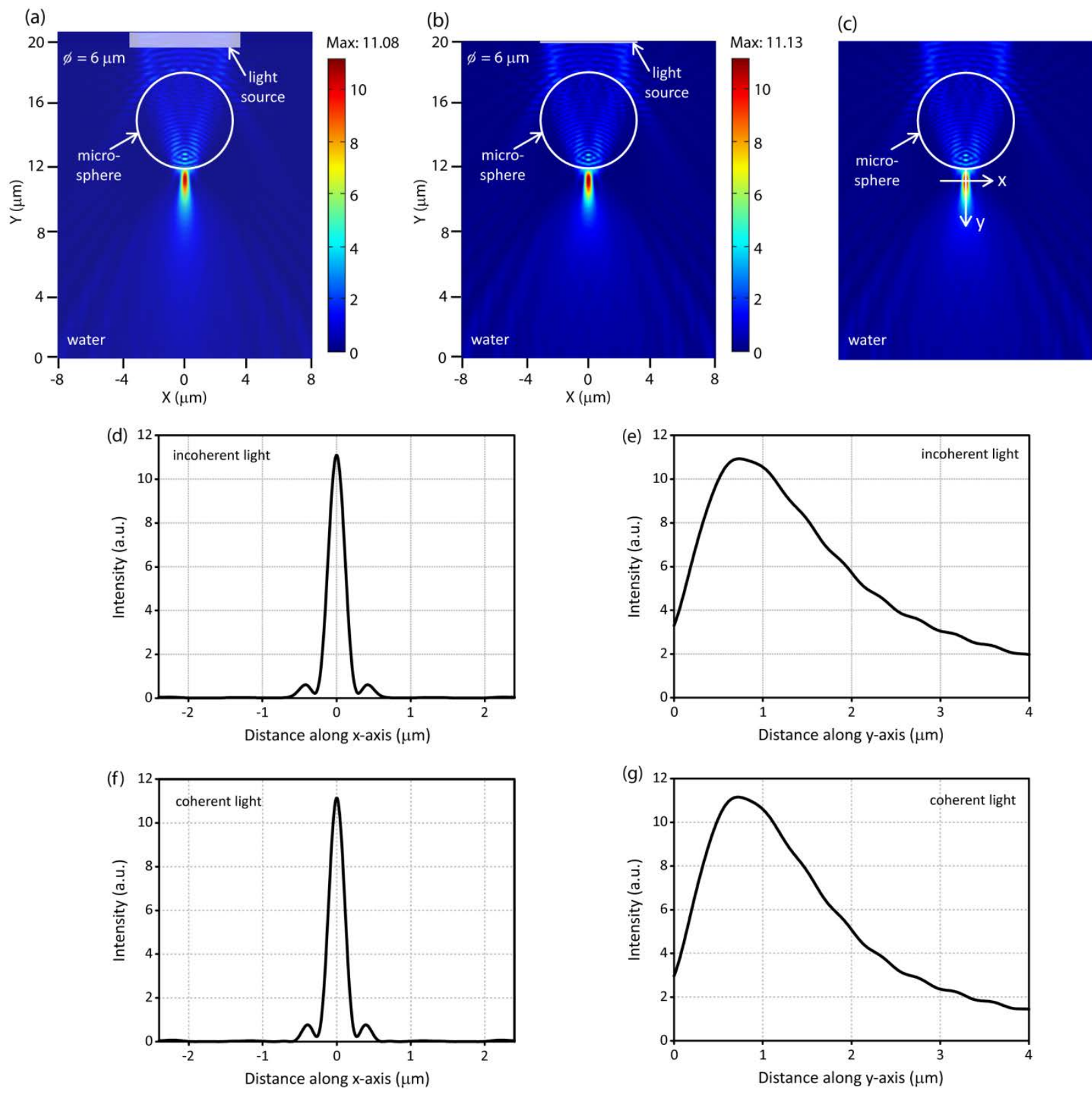

Figure S2. Detail of a Finite Element Method (FEM) simulation of the light intensity distribution in the vicinity of a $6 \mu \mathrm{m}$ microsphere that is placed in water and illuminated from the top. (a) Simulation result obtained by superimposing 11 models with light sources at different positions along the vertical axis. The photonic nanojet can be thought of as being generated under incoherent illumination. (b) The model with a single light source, showing the photonic nanojet generated by coherent illumination. (c) Illustration of the coordinate system used to plot the intensity profiles. The $\mathrm{x}$-axis is along the cross-section of the photonic nanojet waist and its origin is located on the point with maximum intensity in the nanojet; the y-axis is along the vertical direction and its origin is located on the bottom surface of the microsphere where $x=0$. (d,e) The light distribution of the nanojet, as illuminated by an incoherent light source, along the $\mathrm{x}$-axis (d) and along the $\mathrm{y}$-axis (e). $(f, g)$ The light intensity profile of the nanojet, as illuminated by a coherent light source along the $x$ axis (f) and along the y-axis (g). 


\section{S4. Spectrum of the white-light source used in the experiments}

The halogen lamp used in the experiments has a wide-band spectrum, which is from $\sim 400 \mathrm{~nm}$ to $700 \mathrm{~nm}$ and the peak appears at $\sim 600 \mathrm{~nm}$, as shown in Figure S3. Considering the light intensity decreases dramatically when the wavelength is smaller than $400 \mathrm{~nm}$, this part is no longer taken into account for further discussion.

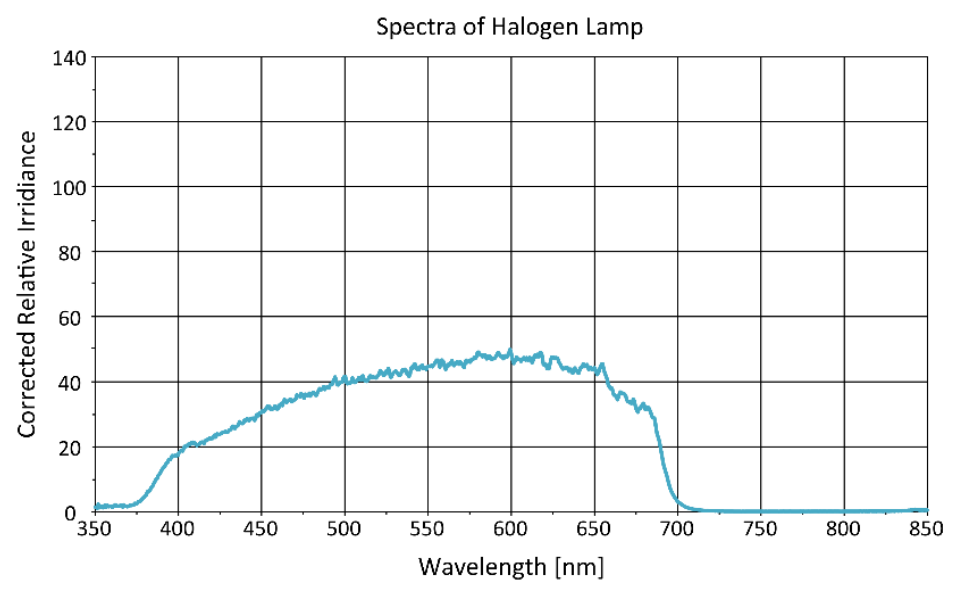

Figure S3. Spectrum of the halogen lamp which is used in the experiments (obtained from Zeiss).

When discussing the relationship between the illumination wavelength and the resolution, the full spectrum of the light source should be considered. The $100 \mathrm{~nm}$ structure can be clearly imaged in the experiments, the resolution is therefore between $\lambda / 4$ and $\lambda / 7$, with $\lambda$ the illumination wavelength in vacuum. According to the Rayleigh criterion, when the sample is immersed in water and the illumination wavelength in vacuum is $400 \mathrm{~nm}$, the minimum feature size that can be resolved by the water-immersion objective with NA $=0.8$ is $188 \mathrm{~nm}$. Therefore, the $100 \mathrm{~nm}$ resolution is clearly obtained by using the microspheres, verifying that our method can achieve super resolution.

\section{S5. Conversion of a near-field evanescent wave into a far-field propagating wave}

From the Maxwell's equations, the wave vector equation for the light propagation in a microsphere with refractive index $n_{p}$ can be expressed as ${ }^{4}$ :

$$
k_{x}^{2}+k_{y}^{2}+k_{z}^{2}=\frac{4 \pi^{2} n_{p}^{2}}{\lambda^{2}}
$$


When the microsphere is simply used as a solid immersion lens, i.e. neglecting the eventual development of the photonic nanojet, for an appropriate size and refractive index contrast, the resolution defined by the classical Abbe’s diffraction limit is:

$$
\Delta_{x-y} \approx \frac{\pi}{\sqrt{k_{x}^{2}+k_{y}^{2}+k_{z}^{2}}}=\frac{\lambda}{2 n_{p}}=\frac{\lambda}{3.8}
$$

Eq. (S3) learns that the diffraction limit is lower in a medium with high refractive index. From our experimental study, an even better resolution than this was obtained (i.e. $\sim \lambda / 4-\lambda / 7$ with $\lambda=400 \mathrm{~nm}$ - $700 \mathrm{~nm}$ ). By comparing this experimental result with eq. (S3), the origin of the super-resolution is suggested: (i) the microsphere first acts as a solid immersion lens and provides local enhancement of the refractive index and a reduction of the effective wavelength, and (ii) the photonic nanojet-related optical path, by which the near-field evanescent waves that carry the fine details of the sample are rapidly transformed into far-field propagating waves, the latter being easily collectable by the microscope.

\section{S6. Effect of phase shift on Gaussian fittings}

As the nanojet arises from wave interferences, both the phase and intensity of the signal should be considered. Here, and as described above, a family of simulated intensity profiles, where the light source is placed at different position along the y-axis, was obtained for a 6 - $\mu$ m diameter microsphere (Figure S4). The intensity profiles at the waist along $x$ of these illumination patterns describing the nanojet profiles were obtained. Note that the vertical shifts $\Delta y$ were chosen to minimize the risk of phase synchronization between the different simulations by ensuring that most of the $\Delta y$ are not multiples of each other. From these profiles, it appears that the phase shift has little to no effect on the intensity profile at the waist.

In an attempt to quantify these variations, these curves were fit with a 1-dimensional Gaussian function $G$

$$
G(x)=\alpha_{G} \exp \left(-\frac{x^{2}}{2 \sigma^{2}}\right)
$$

where $\alpha_{G}$ is a fitting constant. The validity of the Gaussian fitting is discussed below in S7. As detailed in the main manuscript, the value of interest is the standard deviation $\sigma$ which characterizes the quality of the image (labeled $\sigma_{\text {ill }}$ for the illumination profile or nanojet profile considered here) 
and is proportional to the lateral resolution of the imaging system. The computed values for $\sigma_{\text {ill }}$ for all the simulated nanojet profiles, as well as the approximation of an incoherent light source described in S3 are reported in Table S1. As shown from these data, the variation of $\sigma_{\text {ill }}$ is below the smallest mesh dimension ( 22 nm) and can be considered as phase shift-independent. Furthermore, for all the cases considered, the Gaussian fitting was in good agreement with the simulated curves, as shown by the high coefficient of determination $\mathrm{R}^{2}$.
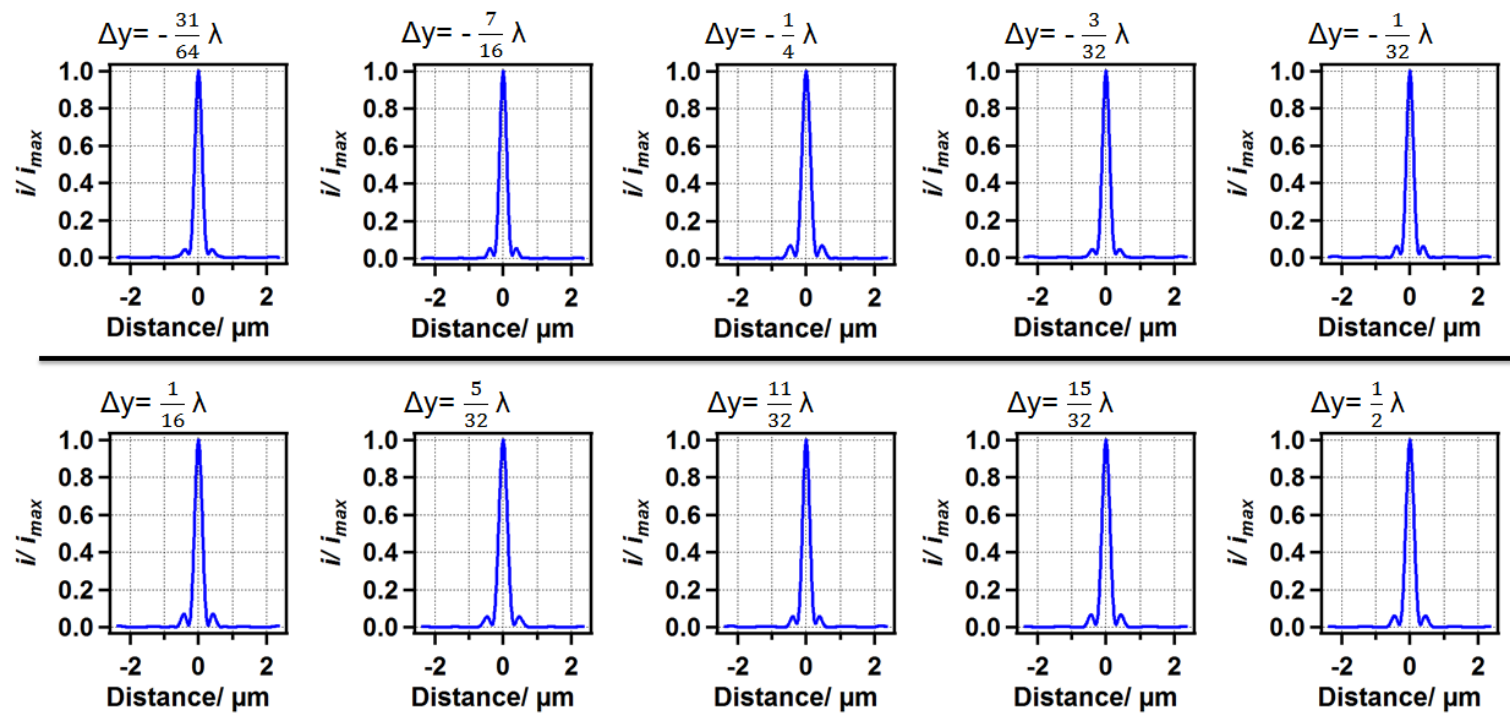

Figure S4. Simulated intensity profiles at the waist of the nanojet, along the $\mathrm{x}$-axis, for a $6-\mu \mathrm{m}$ diameter microsphere and various $\Delta y$.

Table S1. Computed values for $\sigma_{\text {ill }}$ obtained from the Gaussian fitting of the profiles shown in Figure $\mathrm{S} 4$, with the coefficient of determination $\mathrm{R}^{2}$. The results for the simulated incoherent light described in Section S3 are shown in the column 'Sum'.

\begin{tabular}{|c|c|c|c|c|c|c|}
\cline { 2 - 6 } \multicolumn{1}{c|}{} & $\Delta y=-\frac{31}{64} \lambda$ & $\Delta y=-\frac{7}{16} \lambda$ & $\Delta y=-\frac{1}{4} \lambda$ & $\Delta y=-\frac{3}{32} \lambda$ & $\Delta y=-\frac{1}{32} \lambda$ & $\Delta y=0$ \\
\hline$\sigma_{\text {ill }} / \mathrm{nm}$ & 102 & 99 & 116 & 102 & 99 & 100 \\
\hline $\mathrm{R}^{2}$ & 0.998 & 0.999 & 0.974 & 0.998 & 1.000 & 1.000 \\
\hline
\end{tabular}

\begin{tabular}{|c|c|c|c|c|c|c|c|}
\hline & $\Delta y=\frac{1}{16} \lambda$ & $\Delta y=\frac{5}{32} \lambda$ & $\Delta y=\frac{11}{32} \lambda$ & $\Delta y=\frac{15}{32} \lambda$ & $\Delta y=\frac{1}{2} \lambda$ & Sum & $\begin{array}{c}\text { Average +/- } \\
\text { SD }\end{array}$ \\
\hline$\sigma_{\text {ill }} / \mathrm{nm}$ & 105 & 118 & 100 & 108 & 113 & 106 & $106 \pm 7$ \\
\hline $\mathrm{R}^{2}$ & 0.996 & 0.968 & 1.000 & 0.992 & 0.983 & 0.996 & $0.992 \pm 0.011$ \\
\hline
\end{tabular}




\section{S7. Gaussian fittings vs. Airy patterns}

Gaussian fittings are often used to simplify the PSF, especially in the xy-plane. Other functions can be suggested, such as the Airy pattern. This profile $A$ is generated by a wave diffracted through a circular aperture and has the following expression

$$
A(x)=\beta\left(\frac{2 J_{1}(\gamma x)}{x}\right)^{2}
$$

where $\beta$ and $\gamma$ are constants and $J_{1}$ is the Bessel function of the $1^{\text {st }}$ kind of order 1 .
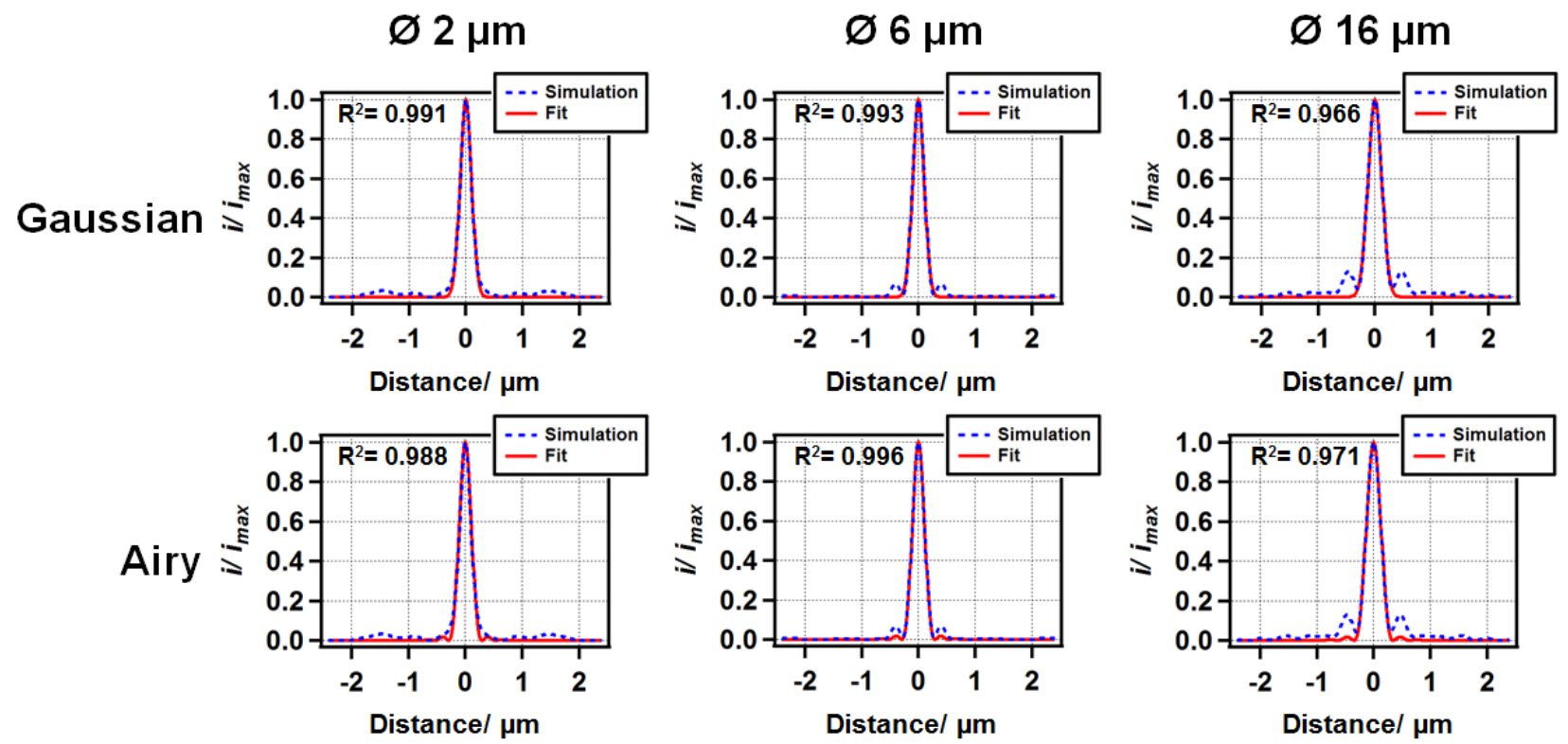

Figure S5. Simulated nanojet profiles taken at the waist, along the $\mathrm{x}$-axis, for $2-\mu \mathrm{m}, 6-\mu \mathrm{m}$ and 16$\mu \mathrm{m}$ diameter microspheres obtained from the simulations shown in Figure 1(b-d). The traces were fit with a Gaussian (top) or an Airy pattern (bottom). The $\mathrm{R}^{2}$ of the fitting is shown in each panel.

The nanojet profiles obtained from the simulations shown in Figure 1(b-d), for microspheres of different diameters, were fit with a Gaussian and an Airy pattern (Figure S5). As detailed in the main text, these curves were chosen, as they are indicative of the profile of the PSF.

Overall, for both the Gaussian and Airy patterns, the fittings were imperfect. Even though the simulated nanojet profile shows a succession of rings, the intensity decays faster for the Airy pattern than for the simulation, hence leading to a poor fit on the additional rings. The observation of the nanojet profiles also reveals that the diffraction pattern generated in the nanojet is not trivial and does not admit an immediate analytical expression, such as a Gaussian profile or an Airy disk. For 
all the fittings, $\mathrm{R}^{2}$ was calculated and is shown in Figure S5. For each of the microsphere geometries, the $\mathrm{R}^{2}$ was comparable for the Gaussian and the Airy pattern. As the Gaussian fitting is easier to derive and introduce in calculations, this expression was chosen to approximate the PSF in the calculations.

\section{S8. Fitting parameters of the line scans}

Several parameters were extracted from the linescans presented in Figure 3 using eq. (5). The variations of $\sigma$ are presented and discussed in the main text, and the results obtained for $x_{2}-\chi_{1}$ and $\alpha$ are shown here in Figures S6(a) and S6(b), respectively.
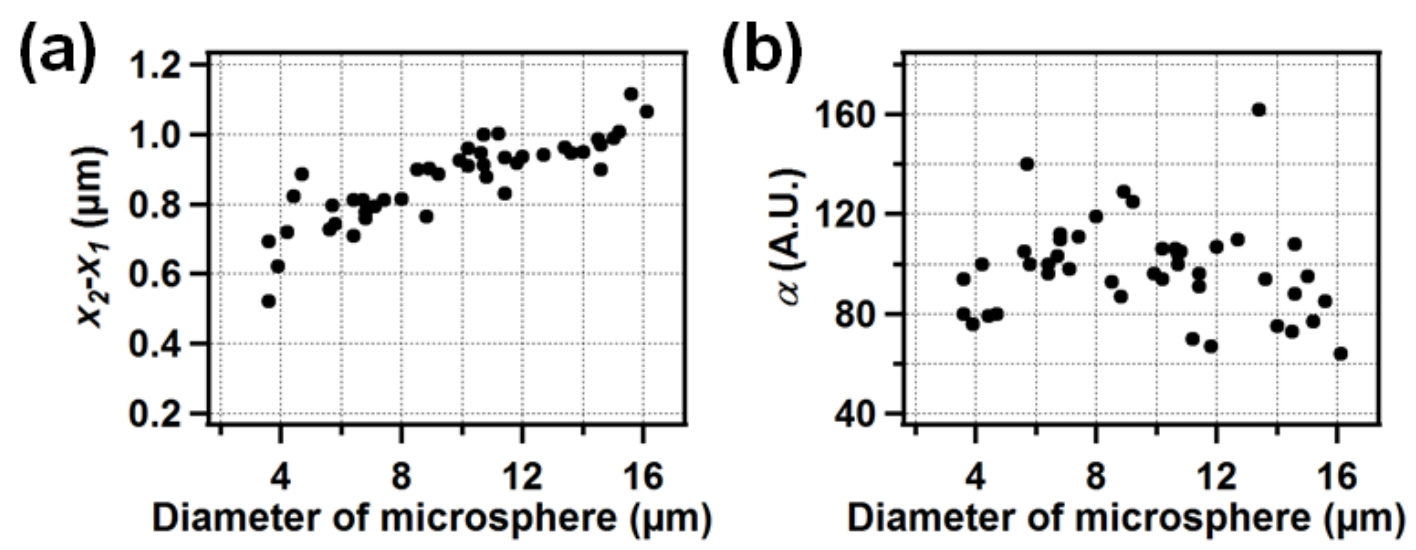

Figure S6. Fitting parameters. The parameters (a) $x_{2}-x_{1}$ and (b) $\alpha$ obtained by fitting the experimental data with eq. (5), as detailed in the main article, are presented as functions of the diameter of the microspheres.

In very good agreement with the sample imaged in this analysis, $x_{2}-x_{1}$ was found to be close to 900 $\mathrm{nm}$ for all the observed microspheres. Indeed, the distance $x_{2}-x_{1}$ between the centers of the two error functions should principally describe the distance between two consecutive gratings, which is 900 $\mathrm{nm}$ in our experiment. Please note that this parameter was here corrected to take into account the magnification. Similarly, the factor $\alpha$ is merely an indication of the variation in intensity. Here, $\alpha$ was found to be largely independent from the diameter of the microspheres, thus indicating that this parameter did not introduce a bias in the fitting and that the maximal and minimal intensities in the different linescans were all comparable, for all the microspheres considered. Overall, these considerations show that this fitting strategy matches the experimental conditions and validate the analysis on $\sigma$ presented in the article. 
Corresponding Author

*E-mail: martin.gijs@epfl.ch

\section{REFERENCES}

$1 \quad$ Parsons, J.; Burrows, C.P.; Sambles, J.R.; Barnes, W.L. Journal of Modern Optics 2010, 57, 356-365.

$2 \quad$ Khoury, C.G.; Norton, S.J.; Vo-Dinh, T. Nanotechnology 2010, 21, 315203.

$3 \quad$ Grosges, T.; Vial, A.; Barchiesi, D. Optics Express 2005, 13, 8483-8497.

$4 \quad$ Yang, H.; Gijs, M. A. M. Microelectronic Engineering 2015, 143, 86-90. 